\title{
Traumatic Wound Microbiome Workshop
}

\author{
B. C. Kirkup Jr. • D. W. Craft • T. Palys • C. Black • \\ R. Heitkamp • C. Li • Y. Lu • N. Matlock • \\ C. McQueary • A. Michels • G. Peck • Y. Si • \\ A. M. Summers $\cdot$ M. Thompson $\cdot$ D. V. Zurawski
}

Received: 9 April 2012 /Accepted: 27 April 2012 /Published online: 24 May 2012

(C) The Author(s) 2012. This article is published with open access at Springerlink.com

\begin{abstract}
On May 9-10, 2011, the Walter Reed Army Institute of Research, as the Army Center of Excellence for Infectious Disease, assembled over a dozen leaders in areas related to research into the communities of microorganisms which colonize and infect traumatic wounds. The objectives of the workshop were to obtain guidance for government researchers, to spur research community involvement in the field of traumatic wound research informed by a microbiome perspective, and to spark collaborative efforts serving the Wounded Warriors and similarly wounded civilians. During the discussions, it was made clear that the complexity of these infections will only be met by developing a new art of clinical practice that engages the numerous microbes and their ecology. It requires the support of dedicated laboratories and technologists who advance research methods such as community sequencing, as well as the kinds of data analysis expertise and facilities. These strategies already appear to be bearing fruit in the clinical management of chronic wounds. There are now funding announcements and programs supporting this area of research open to extramural collaborators.
\end{abstract}

The US military has engaged in active ground combat for the last several years; common patterns of combat injury

B. C. Kirkup Jr. $(\triangle) \cdot$ D. W. Craft $\cdot$ T. Palys $\cdot$ C. Black

R. Heitkamp $\cdot$ C. Li $\cdot$ Y. Lu $\cdot$ N. Matlock $\cdot$ C. McQueary •

A. Michels $\cdot$ G. Peck $\cdot$ Y. Si $\cdot$ A. M. Summers $\cdot$ M. Thompson •

D. V. Zurawski

Department of Wound Infections, Bacterial Diseases Branch,

Walter Reed Army Institute of Research,

503 Robert Grant Ave,

Silver Spring, MD 20910, USA

e-mail: benjamin.kirkup@us.army.mil

B. C. Kirkup Jr.

F. Edward Hébert School of Medicine, Uniformed Services

University of the Health Sciences,

4301 Jones Bridge Road,

Silver Spring, MD 20910, USA include closed head trauma, explosive injury to the limbs, and significant gunshot wounds. As attempts are made to save life and limb, open traumatic wounds are colonized by assemblages of organisms and become vulnerable to serious infections.

On May 9-10, 2011, the Walter Reed Army Institute of Research (WRAIR), as the Army Center of Excellence for Infectious Disease, assembled over a dozen leaders in areas related to research into the communities of microorganisms which colonize and infect traumatic wounds. The objectives of the workshop were to obtain guidance for government researchers, to spur research community involvement in the field of traumatic wound research informed by a microbiome perspective, and to spark collaborative efforts serving the Wounded Warriors and similarly wounded civilians. The two-day meeting included presentations of medium length, lunch presentations, and moderated discussions, interspersed with relatively lengthy opportunities for questions and open discussion. Presenters were given topics. The complete agenda as executed is attached as Table 1, and attendees are listed in Table 2. Not every attendee was able to be present for the entire workshop.

This workshop was critical because no published research has described or addressed the entire suite of organisms colonizing traumatic wounds, despite a significant body of published research investigating chronic comorbid wounds (diabetic ulcers, pressure ulcers, venous stasis ulcers, etc.). Attendees from various government research communities also participated in the discussions. The workshop was supported by the WRAIR commander, funded by the Science Director, and organized by the Department of Wound Infections within the Center of Excellence in Infectious Diseases (Bacterial Diseases Branch). The workshop was recorded, both in audio and video, and the presenters were asked to release the presented materials under a Creative Commons License (attribution). With the exception of a 
Table 1 Agenda as performed

Monday, May 9, 2011

7:00 a.m. to 7:45 a.m.

8:00 a.m.

Breakfast

8:05 a.m. to 9:00 a.m.

9:00 a.m. to 9:30 a.m.

9:30 a.m. to 10:00 a.m.

10:00 a.m. to 11:00 a.m.

11:00 a.m. to 11:30 a.m.

11:30 a.m. to $12: 00$ a.m. 12:00 a.m. to $1: 00$ p.m. 1:00 p.m. to 3:00 p.m.

3:00 p.m. to $3: 30$ p.m. 3:30 p.m. to $5: 30$ p.m.

5:30 p.m. to 7:30 p.m.

Tuesday, May 10, 2011

6:00 a.m. to 8:00 a.m.

8:00 a.m. to 9:00 a.m.

9:00 a.m. to 10:00 a.m

10:00 a.m. to 11:00 p.m.

11:00 a.m. to $12: 00$ p.m.

12:00 p.m. to 2:00 p.m.

2:00 p.m. to 2:30 p.m.

2:30 p.m. to 3:00 p.m.

3:00 p.m. to 5:00 p.m.
Posting of the Colors

Capt. Daniel, MRMC Deputy Commander: Wounded Warriors

Col. Craft: Welcome to the WRAIR

Presentations: Wound Microbiomes: J. Zenilman

Presentations: Traumatic Wound Microbiome: E. Grice

Presentations: The Wound in Context: M. Blaser

Presentations: Exploring Microbial Diversity using Next-Generation Sequencing: M. Sogin

Lunch Discussion: HMP, L. Proctor

Discussion: Genome, Metagenome, Community: Interpreting Complex Data Types in the Clinic

W. Nierman, H. Ochman, C. Liu

Coffee Break

Discussion: Data Tools and Computational Challenges

O. White, L. Price, R. Knight

Dinner

Breakfast

Presentation The Polymicrobial MDRO Infection: D. Relman

Presentation Wound Community Remediation: S. Dowd

Presentation Molecular Wound Diagnostics: P. Murray

Lunch Presentation: Novel Therapeutics and the FDA, MAJ Smith

Brainstorming Structured Collaborations: Col. Craft

Coffee Break

Remarks: Col. McNabb, DBRD

Conversations and Departures
Table 2 Attendees

\begin{tabular}{l} 
Black, Chad \\
Blaser, Martin \\
Craft, David W. \\
Daniel, J. Christopher \\
Dowd, Scot E. \\
Gibbons, Henry S. \\
Glass, John \\
Gordon, Scott \\
Grice, Elizabeth \\
Houchens, Christopher \\
Jagodzinski, Linda \\
Kienker, Linda \\
Kirkup, Benjamin \\
Knight, Rob \\
Leung, Kai \\
Li, Charles \\
Liu, Cindy \\
McNabb, Kevin \\
Murray, Patrick \\
Naik, Ramachandra \\
Nierman, Bill \\
Ochman, Howard \\
Peck, George \\
Petersen, Kyle \\
Price, Lance \\
Proctor, Lita \\
Ravel, Jacques \\
Relman, David \\
Sciarretta, Kim \\
Smith, Kristen \\
Sogin, Mitch \\
White, Owen \\
Zenilman, Jonathan \\
Zurawski, Dan \\
\hline
\end{tabular}

\section{Introduction}

The initial presentations by Capt. Daniel, Deputy Commander, Medical Research and Materiel Command (MRMC), and Col. Craft, Director, Department of Wound Infections, Bacterial Diseases Branch, WRAIR, set the context of the meeting. Capt. Daniel described the context of the MRMC research efforts within the military medical system. Unlike a company that requires a product to sell, the military medical research "business model" includes the patient. Thus, the military jointly focuses on fielded medical materiel and fielded medical knowledge: the answer to military medical needs can be either a product or a doctrine. Each is validated and tested before being put into practice. Medical products are approved by the FDA before being passed to the military clinician; 
fielded knowledge, such as clinical practice guidelines, is passed through peer review and clinical trials before fielding. Capt. Daniel described the research (under the Principal Assistant for Research and Technology) and development arms (under the Principal Assistant for Acquisition) of the MRMC, the several laboratories and their international detachments, and the contracting and advanced development units. These are significant to scientists because the MRMC provides a mechanism to move basic research through advanced development, fielding, and acquisition by the military. The core programs (Military Infectious Disease, Combat Casualty Care, Military Operational Medicine, and Clinical and Rehabilitative Medicine) were each presented briefly, demonstrating the breadth of the MRMC interests and the segments into which the military medical research is conceptually divided.

Col. Craft, Director, Department of Wound Infections at the WRAIR, discussed the clinical and historical context of the meeting. His discussion began with the increase in injuries and wounds in the Iraq and Afghanistan theaters $[1,2]$; moved to the clinical laboratory response to an increase in Acinetobacter isolations reported in MMWR (http:// www.cdc.gov/mmwr/preview/mmwrhtml/mm5345a1.htm); and discussed the epidemiological research that followed, pointing to a nosocomial spread of the Acinetobacter isolated from patients [3]. He also described the five levels of care in the military health care system [4] and the deployed clinical laboratory, including the microbiological test menu and the microbiology augmentation kit for the combat support hospitals. The vision of the Department of Wound Infections with regard to the progression of traumatic wound infections was presented (Fig. 1). Finally, he described the research and policy endeavors in the Department of Wound Infections funded under the Military Infectious Disease Research Program. The Department has substantial breadth in its intramural research program and extramural partnerships, including research on the molecular pathogenesis of specific mutidrugresistant organism (MDRO) nosocomial bacteria, the community of organisms in a wound, and the host response to the infection. Antibiotic usage policies in the military and antibiotic resistance frequency among clinical isolates featured prominently in the question and answer session.

\section{Presentations}

Collectively, the Monday morning presentations brought together the latest published and unpublished insights into the human microbiome in the context of a critical clinical need. Dr. Zenilman, the Director of Infectious Disease at Johns Hopkins Bayview, reviewed the epidemiology, microbiology, and community ecology of chronic wounds on an inpatient service, leveraging his clinical experience and relevant research, while noting the distinction between those and traumatic wounds. In Baltimore, hyperendemic comorbidities such as geriatrics, diabetes, and obesity are closely associated with chronic wounds. Burns are another common clinical presentation which Dr. Zenilman discussed. The wounds associated with these comorbidities include venous hypertension, diabetic, decubitus (pressure), and arterial insufficiency ulcers. These ulcers are particularly long-lasting, are always colonized by bacteria, require debridement, and frequently do not respond well to antibiotic chemotherapy.

Dr. Zenilman posed a broad list of clinical and scientific questions in four areas: descriptive epidemiology, ascertainment methods, etiology, and rational wound management. He particularly focused on the development of rational therapies and clinical practice guidelines. He described the field of chronic wound microbiology as nascent and open.
Figure 1 Traumatic wound infection progression. A flow diagram representing the progression of disease or healing from the time of traumatic wounding through the available stages of infection. Red lines represent the unchecked progression of disease; blue, the progress towards healing; green, the process of successful interventions; brown, the process of largely unsuccessful interventions

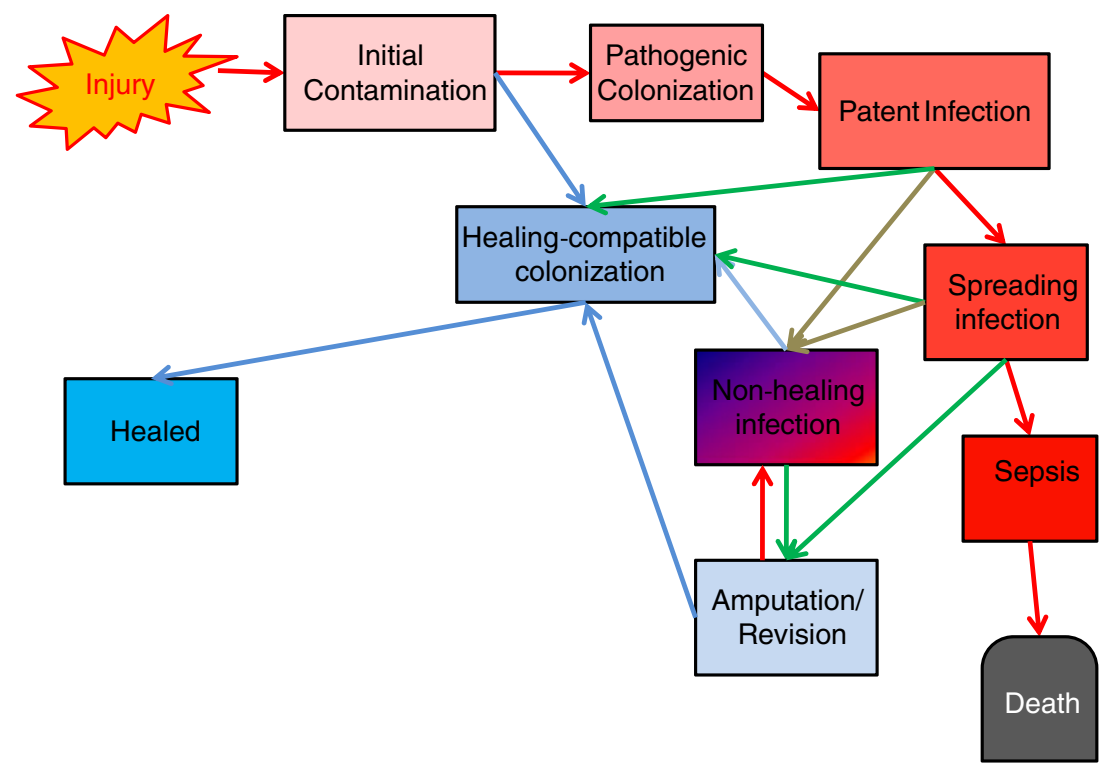


The major clinical practice question raised by Dr. Zenilman was the distinctions among infection, wound healing "arrest," and colonization. In response to attendee questions, he affirmed that there is still no concrete understanding of which bacteria prevent wound healing and how they do so.

The evidence Dr. Zenilman presented was a mixture of clinical photos, sequencing data, culture data, and PCR data. Clinical photos provided by Cmdr. Elster, Naval Medical Research Center, demonstrated the difficulty clinicians face in generating an accurate prognosis of wound healing. Quantitative culture and PCR had each demonstrated that most wounds were polymicrobial, including gram-negative putative pathogens and anaerobes. Sequencing data reinforces the conclusion that the chronic wound flora is always diverse. Fluorescent in situ hybridization refuted the usual objections that raw DNA may be biasing the molecular tests. It also demonstrates the spatial structure of the community. Dr. Zenilman also mentioned the clinical importance of biofilms with regard to antimicrobial resistance, persistence, quorum sensing, and inflammatory deregulation. Finally, he observed that with spatial structure comes the ability to establish different microenvironments, including anaerobic zones. A commonality between chronic wounds and bacterial vaginosis was noted; a joint association via anaerobiosis was hypothesized.

Dr. Zenilman provided detailed guidelines for sampling the wound microbiome based on pilot experiments and personal experience. He discouraged swab sampling and encouraged the taking of a 5-7-mm curette sample (20 mg of tissue) with viscous xylocaine on the leading edge of the wound for clone libraries, quantitative culture, or PCR. Taking these samples did not appear to impact wound healing. Swab results and curette results did not correlate with each other and particularly did not accurately preserve the $10^{5}$ CFU qualitative swab cutoff between "contamination" and "critical colonization" [5]. Sampling location (rim to center, rim to rim) by curette did not change the flora recovered by culture or metagenomics. Swabs are only used clinically by Dr. Zenilman for detecting MRSA.

Dr. Zenilman discussed a broad range of studies, including Maryland ICU surveillance data (active surveillance; Lucy Wilson, culture and PFGE) and a longitudinal study of several burn patients in which antibiotics were used. In a striking fashion, Dr. Zenilman moved fluidly among data collected via clinical microbiology (culture), PCR, and sequencing. This was notable because when close comparisons were made, the results were not usually the same - the organisms identified by culture, sequencing, and PCR are different. In particular, culturing identifies a subset of the population, but no algorithm exists to predict which taxa are overlooked or the phenotypes of those unidentified organisms. The shifting basis for claims - such as statements about the frequency of infections caused by certain organisms, with particular resistance patterns, as compared to statements about not understanding the cause of infections or being able to determine which wounds were in fact infected - passed largely unnoted. Clinicians in particular appeared comfortable with this kind of discourse, bridging the gap between different epistemological strategies and hypotheses regarding wound infections.

Dr. Grice, of the National Human Genome Research Institute, presented her insights into the interaction between cutaneous tissue and the microbiome of traumatic and chronic wounds, derived from animal studies on traumatic and diabetic wounds in a mouse model [6]. During the presentation, she eschewed culture results entirely after an initial slide demonstrating that culture did not capture the diversity of the skin flora. Dr. Grice demonstrated that the normal flora of the genetically diabetic mouse skin is significantly divergent, in quantity and composition, from the normal flora of the heterozygous control mouse. The diabetic mouse skin bears more bacteria overall, especially Staphylococcus. Immediately following wounding, the alpha diversity in the wound quickly declines and then recovers. The wound flora in each mouse begins relatively similar but then diverges (Fig. 2). Dr. Grice interprets this as the wound "selecting" for a certain community. In the normal wound, healing occurs despite a heavy population of Staphylococcus initially, replaced by Clostridia and diverse minor populations. The groups that are apparently underrepresented in the healing wound are the betaproteobacteria and actinobacteria. In the diabetic mouse, the wound becomes chronic. Dr. Grice hypothesizes that this is the result of an inflammatory feedback loop that is proteolytic and oxidative, preventing healing, limiting the natural innate debridement, and leading via re-epithelialization (not contractile healing, which is the primary mechanism of healing in the nondiabetic mouse) to a hyperkeratonic, flakey skin with a deficit of hair follicles and a susceptibility to rewounding. Rewounding leads to a chronic non-healing wound.

The study of healing in a mouse model required some defense precisely because of the role of contractile healing in the mouse, as opposed to the human. Healthy humans primarily re-epithelialize. These differences clearly limit the direct application of the mouse wound model to the evaluation of human wound therapies.

Dr. Grice also discussed the mouse gene expression profiles that accompanied healing in her wound infection model. In the healthy mouse, the gene expression profiles follow the expected pattern of immune activation and deactivation. Key groups of genes include those for the immune response, keratinocyte regeneration, and extracellular matrix production. In the diabetic and non-healing wounds, some genes fail to turn off by day 14; their activation levels mirror the relative abundance of Staphylococci.

Dr. Grice requested that the community provide more support for the analysis of longitudinal data sets; increase 
Figure 2 Comparison of traumatic and diabetic wound progression. A graph demonstrating the difference in wound community composition between wounds in diabetic and nondiabetic mice over a 28-day period

\section{Longitudinal relative abundance of major bacterial groups during healing}

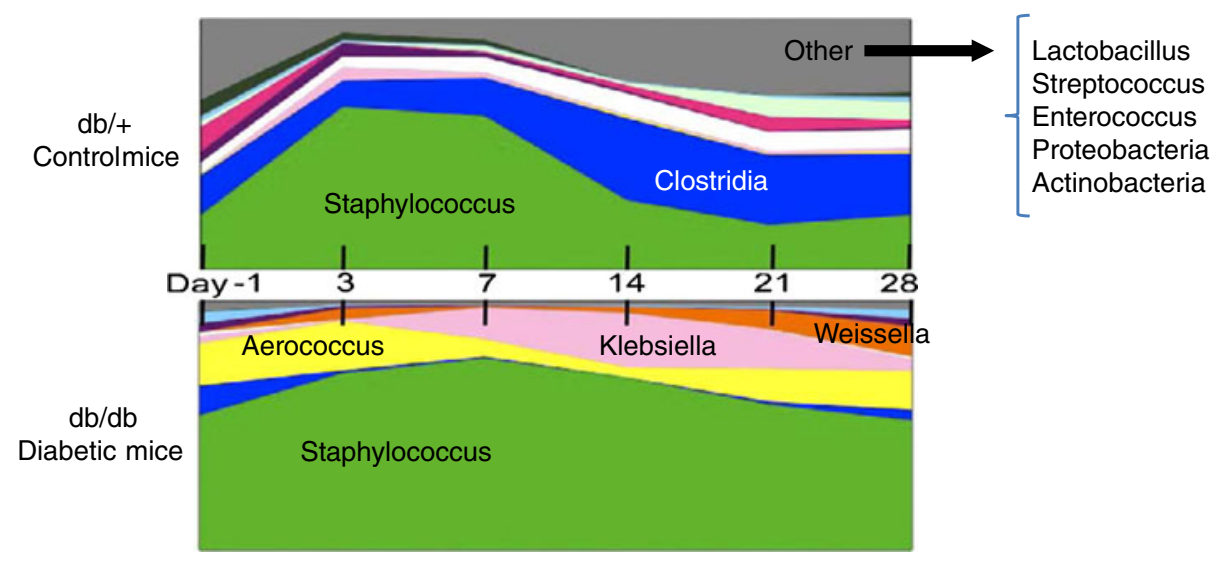

Grice et al. PNAS. 2010. the study of colonization and small-scale microbial migration; and integrate microbial genetic studies with host gene expression studies, particularly with respect to TLR4 and other immune genes.

Dr. Blaser, the Frederick H. King Professor of Internal Medicine at New York University (NYU), provided a wideranging, data-rich presentation of the skin microbiome. Research by groups with which he is affiliated is rapidly translating the basic awareness of the skin microbiome structure into a clinically relevant understanding of pathology by way of clinical investigations. During his presentation, Dr. Blaser provided a focused review of the skin microbiome literature, including interpersonal, intersite and bilateral variation, and the distribution of common bacterial and fungal taxa. He raised the question of skin biotypes $a$ la Arumugam [7] and discussed the results of microbiome studies comparing populations from different communities, including a city in Colorado, New York City, and an Amazonian village.

Dr. Blaser discussed some methodological innovations and concerns. One innovation was the use of eukaryotic DNA quantification to provide a standard allowing the relative quantification of bacterial DNA between samples [8]. Using skin swabs, the variability of the eukaryotic to bacterial DNA concentrations is approximately two orders of magnitude; this may correlate with actual bacterial cell density on the skin surface. The use of different $16 \mathrm{~S}$ rRNA primers was also discussed in some detail; the results from V1-V3 and V3-V5 do not lead to the same conclusions. In presenting a psoriasis project [in collaboration with Barbara Methe of J. Craig Venter Institute (JCVI)], Dr. Blaser presented both the V1-V3 and V3-V5 results without necessarily privileging either one. In later discussion, Dr. Sogin stated that the agreement was actually rather good, and some suggestion was made that the paucity of V1 data in the reference databases was the source of the discrepancy - and would be resolved with growth in the metagenomic databases.

Dr. Blaser discussed several pathogenesis-related studies, including ongoing studies of psoriasis vulgaris and skin abscesses. Both studies are large and complex, requiring multidisciplinary teams to encompass the clinical interface, pathology, microbiology, dermatology, statistics, and informatics. Initial results of the psoriasis study clearly demonstrate the complex interplay of the flora and the immune system in this disease (or diseases). Initial studies on skin abscesses have encompassed some culture-negative infections as well as several seemingly MSSA- and MRSAdominated infections. There are 25 patients enrolled, with age-matched controls. This ongoing study will attempt to understand these abscesses, in particular in relationship to the local skin flora.

Dr. Sogin, of the Marine Biological Laboratory, presented his insights on the long-tailed operational taxonomic unit (OTU) abundance distribution present in many, if not most, natural environments. The long-tailed diversity distribution in microbial ecology is a unifying principle that raises general questions, requires common methodological approaches, and is of practical importance in all environmental engineering applications, including the practice of medicine.

The first question almost always raised by the observation of long-tailed diversity descriptions is whether the observation is accurate. Dr. Sogin reviewed the history of that discussion, with particular reference to Quince et al. [9], Reeder and Knight [10], Quince et al. [11], McClellan et al. [12], and Huse et al. [13]. By way of this review, he presented evidence that the long-tailed distributions of OTU diversity are real and that they comprise $70 \%$ of the diversity in many 
environments (soil, water). Further, he presented methodological innovations which may be missed by the naïve researcher, in particular the use of pyronoise/ampliconoise and chimera detection to clean up sequencing errors and the difficulties with transitive alignments, resolved through preclustering and average neighbor-based cluster building methods to assign sequences to accurate OTUs. He used these methods to analyze experimental data which were mischaracterized using other methods and then applied them to environmental data sets to validate the observations of long-tailed distributions, to determine how best to treat singletons (most appear to be real and cannot safely be discarded as erroneous).

Dr. Sogin briefly discussed the implications of longtailed distributions for ecological theory, including the question of what rare organisms were "doing" in the samples. Recent evidence points to migration from habitats in which the species are common and periodic spikes in abundance for many of the rare taxa; however, other theories, such as keystone species being rare for community structural reasons even in their primary environment, are also supported in some cases. The implications of rare taxa in wound infection diagnostics and therapeutics are still very much unknown.

Finally, Dr. Sogin presented CLASI-FISH, which allows one to interrogate very diverse samples microscopically. By using fluorophores on multiple probes in all possible combinations, dozens, even hundreds, of distinct OTUs can be observed in a single sample. Recourse to microscopy provides a control against sequencing error but also allows observation of community microstructure and a link to cell morphology. This very exciting method and the colorful images produced incited vibrant discussion.

Dr. Lita Proctor, Director of the Human Microbiome Project from the National Institutes of Health (NIH), spoke during lunch. She provided a high-level view of the NIH Human Microbiome Project (HMP; \$175 M program) and many of its early successes, including insights into microbiome diversity, function, and development [14]. The Human Microbiome Project itself has funded foundational initiatives across the field, including the creation of a basic reference genome dataset, the creation of readily accessed data analysis software/pipelines, and the establishment of the Data Analysis and Coordination Center. At the time of the presentation, there were 2 clinical sampling centers, 4 sequencing centers, 9 demonstration projects, 10 computational tools projects, 15 technology development projects, and 5 ethical/legal/social projects funded by the HMP.

Now-classic results produced by the HMP describe the ubiquitous, shared, identifiable, body site specific, age specific, human microbiota. In fact, differences among the microbiota of different body sites swamp the variability derived from other factors such as age, gender, and culture. Among these, she highlighted the gut microbiota as the "cardinal microbiota," (Fig. 3) structured differently among people into discrete types of communities to extract the maximum amount of energy from their environments. Some exciting new data indicate that horizontal gene transfer rates
Figure 3 The cardinal microbiome. A slide illustrating the GI tract and describing the gut microbiome as central among the several humanassociated microbiomes

\section{GI microbiome as 'cardinal' microbiome.}

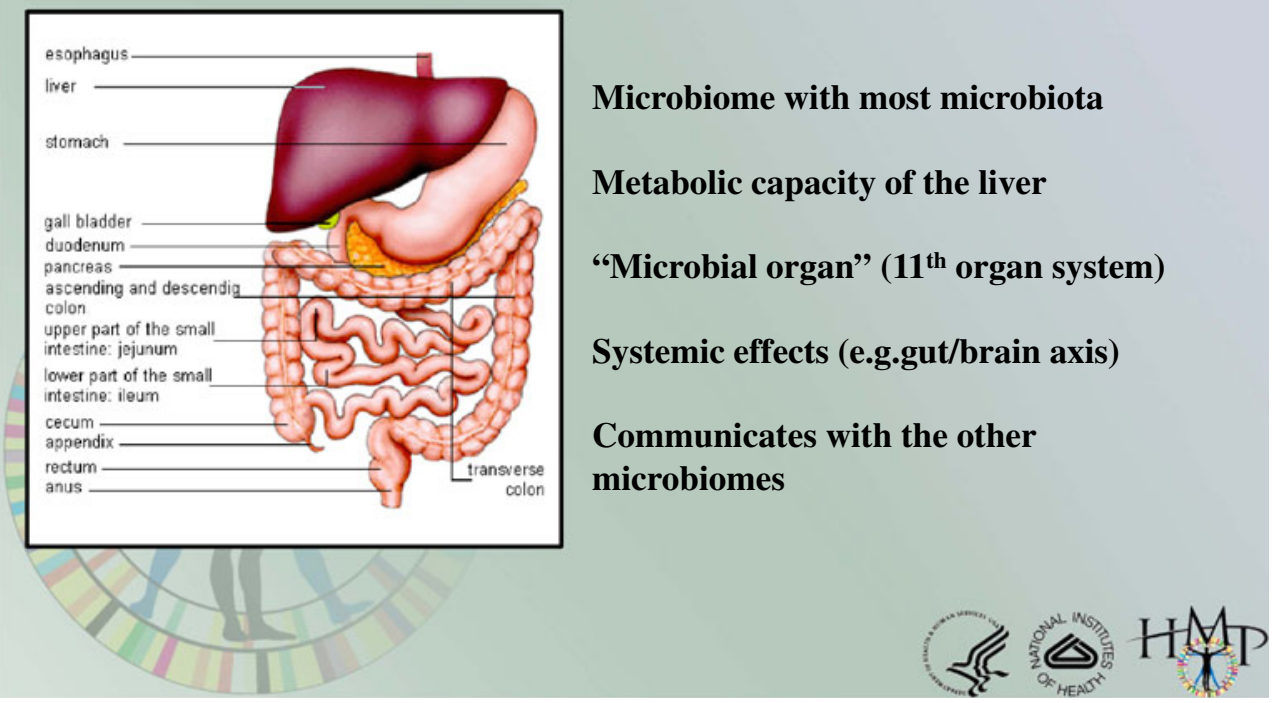


are elevated in the host-associated microbiome relative to air, soil, and marine microbiomes (cited personal communication 2011 from Eric Alm, MIT; now published [15]); this has interesting implications as one might consider megafauna to be spatial hotspots for the generation of genomic diversity within their own habitats. Her major conclusions from the HMP Demonstration projects were that novel disease-associated communities with specific compositions have been described, with both structural and functional genetic markers, and that these findings permit a better understanding of certain diseases as well as the development of new diagnostic tools.

The afternoon consisted of a series of shorter presentations in a matrix of discussions. The presentations were separated into two groups of three. Dr. Nierman of the JCVI presented an overview of the JCVI HMP portfolio, including collaborations with NYU, NIAID, University of Illinois, the Mayo Clinic, Johns Hopkins, McGill, Dow, RTI, Howard University, National Jewish Health, NIDCR, NIDDK, TEDDY, USCD, and Children's National Medical Center; investigating diseases including esophageal cancer, bacterial vaginosis, psoriasis, obesity, colon cancer, asthma, periodontitis, diabetes, UTIs, and febrile illnesses. Despite the number of diseases mentioned, the entire presentation was framed by a review of the normal microbiome and the commensal role of bacteria in human immunity and metabolism (Fig. 4). This is a particularly notable perspective when a disease may be caused by an absence of some microbial colonization or exposure, as opposed to a standard germ hypothesis embodied in Koch's postulates, in which the presence of microbial colonization is the cause of a clinical condition or disease.

\section{Bacterial Species Abundance}

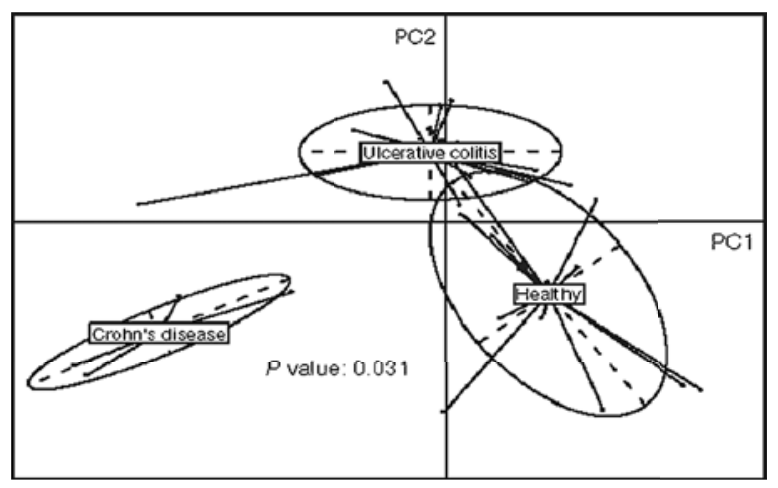

Principal Component Analysis

Qin et al. 2010

Figure 4 Distinctions among healthy and disease-associated flora. A two-axis principal component analysis derived from sequencing elements of the intestinal flora in healthy persons and those suffering from chronic gastrointestinal disease. Clearly the distributions are different, though there is some overlap in these first two principal components between the healthy flora and the ulcerative colitis flora
Dr. Nierman moved quickly to discussing the probiotic notion, which is a joint causal relationship in which a disease is caused by pathogen presence but potentiated by commensal absence; Candida albicans infections, cystic fibrosis (CF), and asthma were all fingered as diseases which may follow this paradigm (naturally, $\mathrm{CF}$ is even earlier potentiated by a genetic problem, Candida infections were created experimentally in gnotobiotic animals, and asthma may have other underlying causes upstream of the alterations in the lung microbiome). The pattern appears to be a decline in diversity followed by invasion by a pathogen.

Asthmatics follow this pattern during their frequent lung infections. Crohn's lesions also follow this pattern [16]. Artificially enriching the populations prior to clinical disease may offer the potential to ameliorate such disorders, but broad application of probiotics requires significant learning about the host system, the commensals, and the pathogen.

Dr. Ochman, of Yale University, delivered a biphasic presentation beginning with his perspective on the state of microbiome studies and ending with a data-rich preview of research being conducted using archived chimpanzee fecal samples. In the initial phase of his presentation, Dr. Ochman distributed a handout listing the current software he uses to manipulate microbiome data; given the audience, this was less an act of information and more an act of demonstration. The point made was that other scientists approach researchers with microbiome experience asking: what should I do with my data? This is unusual because most areas of science do not allow for the successful accumulation of data in the absence of the training to analyze it. However, sequencing technologies have now created a situation in which amplicon sequence data are available; and there is a perception by those not actively engaged in the field that there is one pipeline, one set of tools, and one "answer" while, in Dr. Ochman's words, "nothing is further from the truth." He further emphasized that most "microbiome" studies currently published are actually "microbiota" pattern descriptions and do not provide extensive explanation of the biological processes leading to the observed abundance, diversity, and distribution.

The second phase of Dr. Ochman's presentation described a fascinating project built on the samples collected during prior chimpanzee studies. Of 100 chimpanzees studied, he focused on 35, for which the demographics and genetics are well understood. The chimpanzees' parentage, community affiliation, geography, and diet have been studied already; fecal samples have been collected and stored in freezers. Some samples had been studied in an attempt to understand the origins of HIV. The samples span seasons, migrations, generations, and regions. There are apparently no true "infant" samples which have been studied, but animals of both genders and several ages have been studied over the course of several years. These are habituated animals and have not been treated with antibiotics. 
Preliminary observations revealed first that the chimpanzee hindgut flora is distinct from that of humans and other primates. Surprisingly, there was no detectable signal of host genetic relatedness; geography, gender, community membership, and diet featured prominently in explaining microbiota composition. Migration events were detectable in the data. Further findings are expected as additional samples are processed under Dr. Ochman's "clone by phone" process.

Dr. Liu, of Translational Genomics Research Institute (TGen), presented some insights into the translation of microbiome research to clinical applications. As a clinician, she addressed a communication challenge that faces many putative next-generation diagnostics; that is, the advocates for those diagnostics regularly emphasize difficulty in communicating with the physicians with whom they must continue to interact, and whom will eventually be executing many of the plans and programs which are proposed by practitioners in other communities. The content of her talk emphasized how clinicians are integrated into diverse clinical scenarios and healthcare settings; how they will need to develop either a subspecialty in each area that integrates and interprets genomic, metagenomic, community, ecological, or evolutionary data for the larger specialty, or how a new service will be born, similar to radiology or microbiology, to perform that task for the entire practicing medical community. Decisions like these will interact strongly with the level of complexity that is accepted from the diagnostic instruments and tools themselves; open-ended platforms provide highly complex data, possibly at a significant delay, but with the ability to address unknown or unacknowledged scenarios. These "discovery" systems may be used by teams of specialists, or they may remain in the research laboratories. On the other hand, closed approaches which sort among previously characterized possibilities are generally amenable to direct interpretation by a single specialist or even the clinician himself. As Dr. Liu emphasized, this bypasses complex bioinformatics, sensitivity issues, and zebra chasing.

Dr. Liu made the point that researchers would profit the medical community more by educating physicians and developing systems in partnership with clinicians; more bidirectional learning and interdisciplinary education, and mutual respect. She suggested that genomic medicine, possibly even ecology, should be integrated into the medical curriculum soon, to prepare physicians for the tools of 5 years from now; this includes integrating this material with the board examinations and the gatekeepers that drive "average" medical education today.

A lively discussion followed the talk; one area that received significant discussion was the idea, put forward by Dr. Liu, that some direct physiological measurements might be more useful than genetic or genomic data. Dr. Proctor suggested using serum as an integrating signal, a form of metabolomics. One idea presented was that therapeutics could be piloted ex vivo before being introduced into patients. Dr. Sogin registered skepticism because of the time frame (missing ecology and evolutionary potential) and the lack of biological context; Dr. Gancz suggested that such a model would at least provide another term in the approximation for the real system by moving beyond pure cultures to consortia, embracing signaling and co-metabolism on a short time scale. Dr. Proctor reminded the group of the long history environmental engineers have using black box microbial assemblages and how they are related to the current fecal transplant research and brainstorming conducted by Linda Chrisey and her team at the Office of Naval Research. Dr. Knight introduced some research results suggesting that transplanting new functionality into a gut by transplanting flora did not succeed when the goal was to allow mice to degrade cellulose in conjunction with termite flora.

Dr. White, of the Institute for Genome Sciences, University of Maryland Baltimore School of Medicine, is the principal investigator at the Human Microbiome Project Data Analysis and Coordination Center (DACC). He presented his vision for how the DACC can serve the needs of the wound microbiome research community. His statements about the data-intensive nature of microbiome research reinforced the experience of all the active researchers in the room. The HMP presents a salient case of what has been popularly called the data tsunami. The DACC deals with a "scary" data management problem, assembling vast quantities of data from numerous sources into one mutually informative warehouse for the research community. No single researcher has the persistent capability to generate, process, store, and analyze his own data robustly; some researchers specialize in sample acquisition, others in sequencing, while still others produce software pipelines to perform the analysis. Many times these researchers are not even members of the same groups, institutions, or even research communities. The DACC provides interfaces between datasets, software utilities, and computing resources to allow researchers to access the products of other research groups without undue friction. It also collaboratively produces the standards, documents, SOPs, and workflows required to support inter-institutional collaborations.

The HMP has made substantial use of the DACC. Unlike many prior projects which could have benefitted from a similar center but did not, the funding to establish the DACC was designed into the original HMP scope of project - and the utility of the DACC will extend long beyond the end of the initial HMP. The goal is a system which allows meaningful comparisons among data sets generated for different purposes, in a scalable, elastic, accessible, persistent, and efficient form. Dr. White sees the path to this goal as leading through the development of the Open Science Data Framework, which provides flexible interfaces (APIs) to underlying "infrastructure as a service" and, ultimately, "data as a service." 
Realizing this vision requires relatively rigid standards to manage data types and processes, especially when the underlying technologies and algorithms are constantly shifting. The distinctions between data generation technologies (sequencing technologies, for example) are generally acknowledged, and their various merits are often debated. In addition, the algorithms used in each stage of the data analysis may appear similar but conceal critical distinctions. Many groups are currently contributing software to the microbiome community; several are also serving as aggregators and developing architectures to perform integrated data analyses. Examples of software packages which are currently available and being supported are CLoVR [17], MG-RAST [18], and Quantitative Insights Into Microbial Ecology (QIIME) [19]. These packages each provide pipelines that vertically integrate different analysis tasks, from cleaning up sequence data through assigning operational taxonomic units to generating dynamic visualizations of the data.

In the vision presented by Dr. White, rigorous agreements about data types (both experimental and intermediate analytical results) are the structure on which the entire community can hang its own contributions. These agreements are generated by consortia of active researchers and supported by standards and documentation. Data flow into a common data store and can be analyzed through different shared algorithms and viewed in various aspects to answer distinct questions. Intermediate calculations - particularly intensive ones, such as genome assembly — can be stored centrally to support these various analytical strategies (termed "reusable results"). At the same time, competing algorithms which reach similar classes of results can be applied in parallel, as they suit different needs in the community.

This vision is one of a mature community and field of endeavor with common kinds of questions and unique resources pooled in a novel way. Dr. White presented some technical information about the current and near-term state of affairs, including the intervening months between the presentation and this publication, but ultimately he was conveying a sweeping view of the road ahead and suggesting the scope and scale at which the community can come together to support common methods and resources.

Dr. Price of the TGen introduced the material and digital systems which TGen is developing to manage complex genetic, genomic, and metagenomic research and clinical programs. The material systems include kits (bar coded) sent to sample providers, a sample repository, a "biorefinery" (laboratory for specimen preprocessing), and sequencing/data collection facilities. Software mirrors this structure with modules for specimen tracking, clinical information management, laboratory information management, and genomics information management (the BMS, CIMS, LIMS, and GIMS) all built on a data warehouse (Bio4D) and tied to an interactive data portal. The system is portable, built on Java, and compliant with data security and information assurance standards. It is already being used in studies, including one on the microbiota of burn patients. The software is not currently freely distributed, but according to Dr. Price it may be licensed from TGen.

Dr. Knight, of the University of Colorado, finished the first day by discussing analytical tools as applied to microbial community data. His focus on visualization tools provided a graphically engaging presentation. The underlying package he employed is QIIME (free to the public), an integrated pipeline which allows the analysis and visualization of 16S rRNA sequence data from mixed bacterial communities, metagenomics data, and, with some tweaking, related data types (for instance, functional gene sequences).

Driving the need to analyze and visualize vast amounts of sequence data is the decline in sequencing costs. In the Knight Laboratory, sequencing costs are now eclipsed by DNA extraction as the major cost behind community analysis, in part because Illumina is the primary sequencing technology employed [20], with barcoded samples. The expected cost of sequencing 96 samples for $16 \mathrm{~S}$ rRNA genes is expected to fall to $\$ 1,000$ with the advent of the GS Junior, dropping below the costs of laboratory preparation.

Perhaps in contrast to some of the published literature and even other speakers, Dr. Knight was unequivocal that the cost of data analysis is not a major issue. Using QIIME to perform the kinds of analyses he presented is computationally inexpensive; unlike de novo genome assembly, for example. Given the low cost of sequencing and the low cost of data processing, the remaining issues tend to be sample collection, hypothesis generation, and experimental design - in short, the hard work of science.

Sample collection as a difficulty can be split into two parts: sample management and metadata collection. Most researchers have less difficulty than does Dr. Knight with sample collection; however, he discussed his Earth Microbiome Project, with a current expected cache of 40,000 samples and an eventual goal of processing 200,000 samples. These samples, along with the Human Microbiome Project samples and other samples from other studies, are meant to allow a researcher to view their own study samples in the context of a universe of other samples. This presents challenges. From a sample management perspective, Dr. Knight discussed working backwards from the microbial sequence data to identify a mislabeled sample. On the other hand, the data that come along with the samples are critical; Dr. Knight discussed the difficulties in acquiring good metadata and finding well-structured databases of clinical data. The spread of good database practices and metadata standards (such as well-managed electronic health records) would do much to advance the field of microbial ecology and to allow its direct application to human health. 
The studies Dr. Knight presented included the biogeography study of keyboards and fingertips, the geography of the face, a time-series study of several individuals at 27 body sites, and a time-series analysis in which mice bearing humanized gut flora were fed different diets to monitor weight gain. Principal coordinate analysis featured prominently as an analytical tool, and supervised classification techniques are now becoming prominent in the Knight Laboratory. Most striking in the presentation and in some recent publications, videos which allowed an additional dimension to be visualized were both revealing and convincing; as always, data presentation is a rhetorical art.

Dr. Relman, of Stanford University, used an ecological framework to provide a theoretical framework for understanding wound infections. He explicitly integrated island biogeography into the discussion, including assembly rules, migration rates, and inter/intraspecific competition/cooperation. This biogeography has been best explored at the level of the individual, particularly during the primary succession events of the infant gut. This critical succession may be one of the only primary successions which occur on a spatial/ temporal scale similar to the events following a major traumatic injury or critical burn. Research by Palmer et al. [14] and Dominguez-Bello [21] has described the opportunist exposures that lead to the early flora, such that some infant gut microbiota most resemble maternal gut flora, others maternal vaginal flora, and still others, maternal skin flora-in part due to the birth process, which differs between vaginal birth and surgical birth. Recent publications suggest that this infant gut flora may have long-term health implications for the individual, giving direct clinical relevance to research of this kind [22].

At a very different scale, Dr. Relman also discussed the repeatable distinctions among the microbiota populating different facets of individual tooth surfaces [23]. On work of this scale, the quality of the sequence was absolutely critical. Each site was found to be a nonrandom subset of the mouth diversity. There was no obvious lateral symmetry, though disease tends to be symmetric. Migration appears to play very little role in populating tooth surfaces.

No study of landscape ecology would feel complete without a discussion of anthropogenic meddling; filling this important trope for wound ecology is a discussion of antibiotic treatment. The discussion launched from the work of Jernberg [24] and Dethlefsen [25, 26], highlighting the long-term impact of antibiotic treatment, the impact of repeated antibiotic treatment, and the question of community resilience or lack thereof. The general conclusion was that there were long-term effects; that repeated shocks increased the impact of the perturbation such that resilience was lost before the community composition changed radically; and that based on functional gene studies, there were likely to be biochemical functions impacted in the later shifts, though phylogeny seemed to change more than biochemistry did.

Ultimately, the translation of the methods of biogeography into the study of traumatic wounds brings a rich tradition of questions, tentative answers, debates about theory, results, and conclusions into a clinical setting. It raises questions about assembly rules, assemblage composition, community function, ecosystem services, resilience, and ultimately the response of the whole (eukaryotic and microbial) host; and provides strategies to approach their answer through natural and anthropogenic experimentation in the same way that island biogeography and landscape ecology have been explored, particularly in the five decades following McArthur's pioneering work.

From this perspective, Dr. Relman concluded that though the community is collecting vast amounts of microbiome data, deficiencies in study design may compromise its value. He emphasized the need for carefully managed time courses and structured metadata, a theme that recurred elsewhere.

Dr. Relman's clinical experience provided an interesting counterpoint to his research perspective. From a research perspective, he discussed the complexities of bacterial communities, antibiotic resistance [27-29], and the blinkered view inherited by being informed solely with culture results; but from the clinical perspective, in the short term, his recommendation is the studious application of clinical art to provide focused treatment to wounds with certain organisms, to not cover everything, to ignore even "recognized" and antibiotic-resistant "pathogens" depending on clinical context and instinct. In particular, he recommended learning from past experience and avoiding an antimicrobial game of "whack-a-mole." This very practical advice lent an earthy tone to a talk otherwise deeply flavored by theory.

Dr. Dowd, currently with MR DNA aka Molecular Research LP., spoke about his experience with chronic wound diagnostics and therapeutics. His diagnostic group and the associated clinic treat non-healing wounds such as diabetic, decubitus, pressure, and vascular insufficiency ulcers, particularly infections that present limb, even life, risks. At the time the practice opened, these wounds led to an amputation rate of $100 \%$.

The current therapeutic strategy at the clinic centers on debridement as the primary means of removing bacteria and dead tissue [30-32]. Repeated debridement alone has a significant positive impact on healing, particularly when combined with the correction of systemic host factors (such as blood glucose management). Besides this universal approach to wounds, the clinic and diagnostic laboratories use molecular methods to determine the microbiota of each wound [33], which inhabits the wound in a structured community [34]. The therapeutic process that derives from the molecular diagnostics includes local antibiotics applied within a 3-day window of the debridement [35] to prevent reestablishment of the 
community. The current delivery mechanism is a lipogel [36] which releases antimicrobials into the wound.

Molecular methods change the diagnosis of many infections [33]. Wounds that provided no culturable bacteria often present numerous bacteria, including some expected to be culturable (such as Klebsiella). Wounds which present a single culturable organism instead present a complex community. Many genera are missed frequently by culturing even when numerically dominant in a wound. Streptococcus are the second most common major wound organism; Morganella, Finegoldia, Corynebacteria, and a range of anaerobes including Bacteroides, Anaerococcus, Peptoniphilius, Clostridium, and Actinomyces are also common. Naturally, Staphylococci, Enterococcus, Serratia, Proteus, enteric bacteria, Acinetobacter, Providencia, Pseudomonas, and yeast are still well represented. Some organisms are frequently misidentified, including Peptoniphilus harei, Corynebacterium striatum, and Prevotella. Ultimately, $86 \%$ of the genera identified by molecular methods from a suite of chronic wounds could be identified in clinical culture as well, when the laboratory used sampling methods specific to anaerobes and used several media and enrichment environments over a period of 4 weeks. However, surgical site infections posed a unique problem; frequently, the Bacteroides which dominated those wounds required co-growth in a polymicrobial wound biofilm model [37]. In $99 \%$ of wounds, $1 \%$ of the population is easy to grow and is characterized by routine clinical cultures [38].

As a result, the clinical diagnostics performed at the wound care clinic substitute molecular methods for culture. The initial method targets 27 organisms by qPCR and provides an answer in $24 \mathrm{~h}$. Every 6 months, additional targets are added to the qPCR assay. A second-level molecular assay is driven by sequencing and reports bacterial identification results in $48 \mathrm{~h}$.

Molecular assays for fungi (18 s sequencing) report that $23 \%$ of chronic wounds contain significant fungal populations, while typical literature rates of fungal co-infection are 2-3\% [39]. Additional co-infecting organisms include algae and Cyanobacteria in certain patients. These do not seem to inhibit healing. Other organisms not commonly treated in wound clinics include some which apparently do need to be treated, particularly Corynebacteria.

A retrospective study of 1,300 patients concluded that wound management techniques at the clinic have improved since 2007. Using culturing as a diagnostic tool appears to impose a $30 \%$ penalty on time to heal. Focusing on the worstcase patients, in the top $10 \%$ of time to heal, healing time has progressed from 150 days without molecular diagnostics or topical antimicrobials to 62 days with only molecular diagnostics, and finally to 37 days with topical antimicrobials. These statistics brought the talk around from the initial images of debridement and patient infection to a more positive and encouraging finale.
Dr. Murray, then-head of the Clinical Microbiology Service in Department of Laboratory Medicine of the NIH, spoke on the future of diagnostic wound microbiology. He discussed the core of traditional wound diagnostic microbiology: microscopy, culture, susceptibility testing, and strain typing. Innovations have been improving each of these, but they continue to show serious weaknesses. Microscopy provides limited, presumptive identifications and no sensitivity data. Culture methods have many flaws which have been discussed at length elsewhere. Susceptibility testing and biochemical identification rest on a shaky foundation of culture, are less accurate than normally acknowledged, and are slow. Finally, strain typing methods - even MLST — have had serious flaws revealed by whole genome sequencing; but sequencing itself presents serious challenges, particularly with assembly and interpretation.

Dr. Murray's laboratory at the NIH has been investigating a range of additional closed- and open-ended technologies, including qPCR, microarrays, gene sequencing, whole genome sequencing, and proteomics. The laboratory has a significant number of qPCR assays in support of research protocols. In addition, gene sequencing (16 s rRNA, ITS, and $\mathrm{Sec}$ ) are used to provide identifications for uncommon and slow-growing organisms. In particular, Nocardia and Mycobacteria can be identified overnight directly from acid fast-positive specimens. Filamentous fungi, which can easily be misidentified by microscopy alone, are also identified by sequencing.

At Dr. Murray's clinical laboratory, microbial whole genome sequencing is already used for certain outbreak analyses (e.g., multidrug-resistant Acinetobacter baumanii). It is particularly valuable when other methods of phylogenetic reconstruction are discordant.

Proteomics, in particular MALDI-TOF, is a particular favorite for routine bacterial identifications because of the speed and efficiency of the method. As the database grows, the concordance with the gold standard identifications increases steadily. At this time, it is at $98 \%$ for bacteria and yeast species [40-44]. Even the difficulties are now predictable; foreseeable, reliable difficulties can be circumvented.

The method holds further promise for two reasons. First, samples can be prepared directly from positive blood cultures [45]. Secondly, there may be more information to be gained from the same assays because the data currently utilized are only a fraction of the available signal, albeit that fraction with the lowest confounding noise relative to the gold standards of identification. Using more of the peaks which come from the TOF MS may allow for additional information such as strain typing to be gleaned from the same process.

In the immediate future, Dr. Murray expects gene sequencing to be displaced by proteomics and strain typing to move to whole genome sequencing. Pathogenicity estimation may also 
be addressed by these two methods. Deep sequencing of communities will be used for quantification of organisms in complex assemblages and pathogen discovery. Metabolomics has potential to address questions of drug resistance, metabolic activity, and virulence.

MAJ Smith, the Director of the Clinical Services Support Division, U.S. Army Medical Materiel Development Activity (USAMMDA), ended the talks by discussing the function of USAMMDA in supporting medical research and product development. USAMMDA currently has approximately 70 open applications with the FDA and provides an interface between researchers, the military, and the FDA. It supports diverse products including drugs, devices, prebiotics, vaccines, and any other regulated product. Her presentation emphasized the need to address regulatory concerns early in product development, particularly for FDA-regulated drugs, devices, and biologics. She presented the nuts and bolts of how the Army provides support to medical researchers as they explore potentially fruitful concepts and later transition their products to clinical trials and advanced development (Fig. 5). In particular, addressing regulatory concerns early keeps researchers out of trouble, limits rework, and enables easy transitions to manufacturing. USAMMDA offers significant experience and support to researchers so that they need not encounter the FDA alone or unprepared. She suggested consulting USAMMDA for any militarily relevant medical research effort prior to candidate selection (vaccine components/raw materials; show-stopping issues like Ames scores; testing requirements), while planning manufacturing, while developing protocols for preclinical safety, and during preIND/IDE planning. She gave some general advice on product development and reminded the attendees that the US Military only uses medical materiel under strict regulation; typically only FDA-approved materials are used in medical care. The regulation quoted was: "Personnel carrying out military operations shall be provided the best possible medical countermeasures to chemical, biological or radiological warfare or terrorism and other health threats. The DoD Components

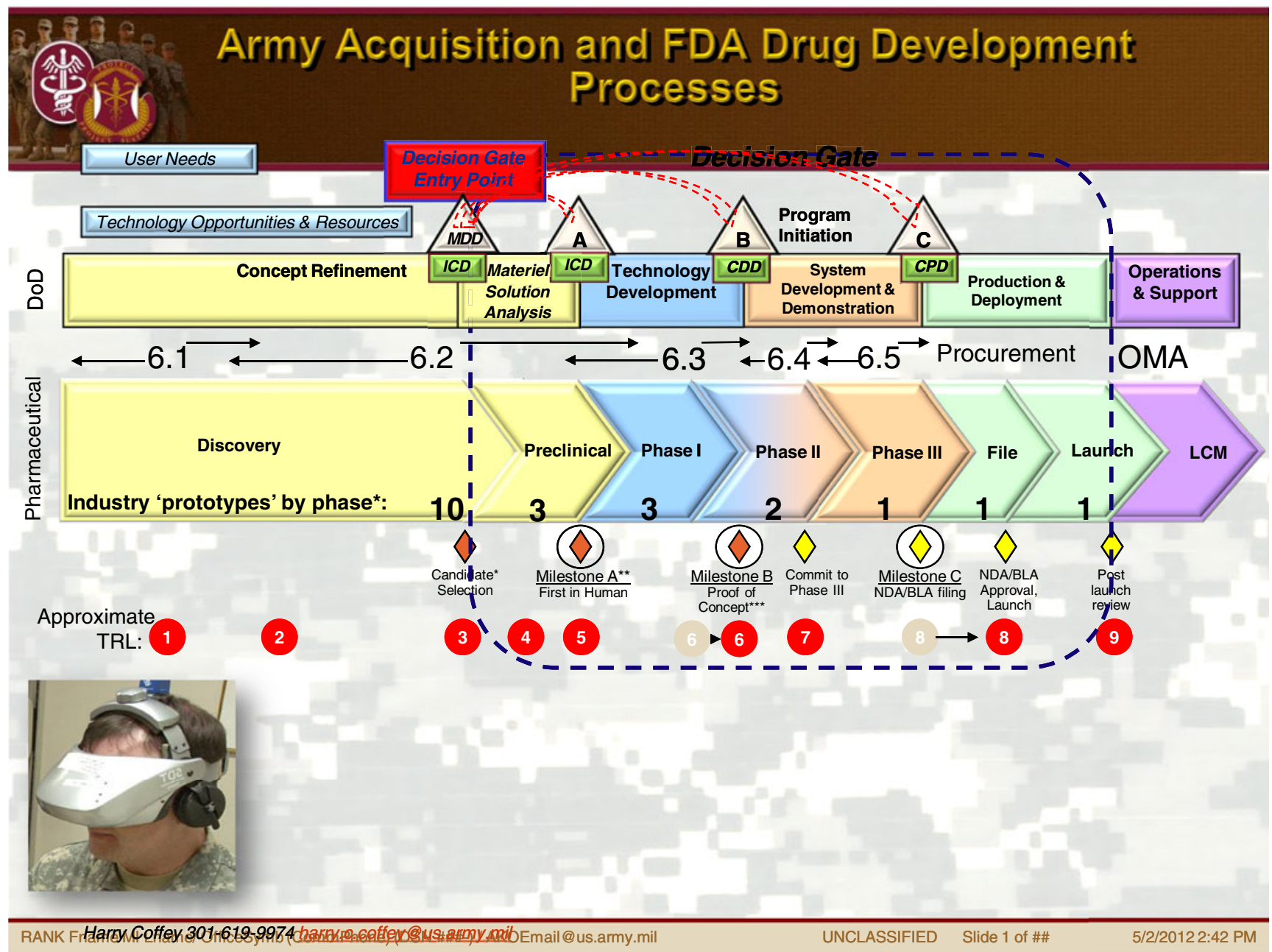

Figure 5 Synchronizing drug development among industry, the FDA, and the DoD. This slide illustrates the different terminology and classifications used to describe progress along the developmental trajectory of a new drug. Each organization has distinct language and key offices assist in liaison and coordination, without which the interfaces can be treacherous 
shall make preferential use of products approved by the FDA for general commercial marketing, when available, to provide the needed medical countermeasure." In addition, though USAMMDA is available to assist researchers, the Department of Defense does not get special consideration or exemptions from FDA regulations. Rather, it carries the additional burden of complying with Federal and Department of Defense Acquisition Regulations. The Major's talk was a fitting conclusion to a series of presentations very much focused on the hope of new diagnostics and therapeutics for the treatment of combat-related wound infections.

Col. McNabb, Director of Bacterial Diseases at the WRAIR, closed the conference with comments about the collective need for a collaborative strategy to include researchers and clinicians. Following his comments, the group discussed potential collaborations before dispersing. Several of the attendees already participate in the Human Microbiome Project and have longstanding collaborations. In particular, the community centers around several sequencing centers, software development groups, and data analysis centers which were represented at the workshop. The opportunity to introduce more people into this community was one of the benefits derived from the meeting.

\section{Conclusions}

The workshop was 2 days of intensive and wide-ranging discussion. Participating government researchers obtained guidance, and the research community was encouraged to become involved in the field of traumatic wound research. During the discussions, it was made clear that the complexity of these infections will only be met by developing a new art of clinical practice that engages the numerous microbes and their ecology. It requires the support of dedicated laboratories and technologists who advance research methods such as community sequencing, as well as the kinds of data analysis expertise and facilities represented by the HMP DACC and TGen's Bio4D system. These strategies already appear to be bearing fruit in the Research and Testing Laboratory, where the clinical management of chronic wounds has been improving steadily. There are now funding announcements and programs supporting this area of research open to extramural collaborators.

Acknowledgments The Command of the WRAIR, particularly Col. KE Kester, MC, and the Science Director, Dr. MT Vahey, is acknowledged for supporting and financing the workshop. Spc. Saini and Spc. Fly are acknowledged for their logistical support during the conference.

Disclaimer Material has been reviewed by the Walter Reed Army Institute of Research. There is no objection to its presentation and/or publication. The opinions or assertions contained herein are the private views of the author, and are not to be construed as official, or as reflecting true views of the Department of the Army or the Department of Defense.
Open Access This article is distributed under the terms of the Creative Commons Attribution License which permits any use, distribution, and reproduction in any medium, provided the original author(s) and the source are credited.

\section{References}

1. Lounsbury DE, Brengman M, Bellamy RF (eds) (2004) Emergency war surgery. Borden Institute, Walter Reed Army Medical Center, Washington, DC

2. Peoples GE, Jezior JR, Shriver CD (2004) Caring for the wounded in Iraq-a photo essay. N Engl J Med 351:2476-2480

3. Scott P, Deye G, Srinivasan A, Murray C, Moran K, Hulten E, Fishbain J, Craft D, Riddell S, Lindler L, Mancuso J, Milstrey E, Bautista CT, Patel J, Ewell A, Hamilton T, Gaddy C, Tenney M, Christopher G, Petersen K, Endy T, Petruccelli B (2007) An outbreak of multidrug-resistant Acinetobacter baumannii-calcoaceticus complex infection in the US military health care system associated with military operations in Iraq. Clin Infect Dis 44:1577-1584

4. Craft DW, Horn GV, Kallstrom G, Bowden R, Mahlen SD (2010) Practicing clinical microbiology in austere environments. Microbe 5:297-303

5. Frankel YM, Melendez JH, Wang NY, Price LB, Zenilman JM, Lazarus GS (2009) Defining wound microbial flora: molecular microbiology opening new horizons. Arch Dermatol 145:1193-1195

6. Grice EA, Snitkin ES, Yockey LJ, Bermudez DM, Liechty KW, Segre JA (2010) Longitudinal shift in diabetic wound microbiota correlates with prolonged skin defense response. Proc Natl Acad Sci U S A 107:14799-14804

7. Arumugam M, Raes J, Pelletier E, Le Paslier D, Yamada T, Mende DR, Fernandes GR, Tap J, Bruls T, Batto JM, Bertalan M, Borruel N, Casellas F, Fernandez L, Gautier L, Hansen T, Hattori M, Hayashi T, Kleerebezem M, Kurokawa K, Leclerc M, Levenez F, Manichanh C, Nielsen HB, Nielsen T, Pons N, Poulain J, Qin J, Sicheritz-Ponten T, Tims S, Torrents D, Ugarte E, Zoetendal EG, Wang J, Guarner F, Pedersen O, de Vos WM, Brunak S, Dore J, Antolin M, Artiguenave F, Blottiere HM, Almeida M, Brechot C, Cara C, Chervaux C, Cultrone A, Delorme C, Denariaz G, Dervyn R, Foerstner KU, Friss C, van de Guchte M, Guedon E, Haimet F, Huber W, van HylckamaVlieg J, Jamet A, Juste C, Kaci G, Knol J, Lakhdari O, Layec S, Le Roux K, Maguin E, Merieux A, Melo Minardi R, M'Rini C, Muller J, Oozeer R, Parkhill J, Renault P, Rescigno M, Sanchez N, Sunagawa S, Torrejon A, Turner K, Vandemeulebrouck G, Varela E, Winogradsky Y, Zeller G, Weissenbach J, Ehrlich SD, Bork P (2011) Enterotypes of the human gut microbiome. Nature 473:174-180

8. Gao Z, Perez-Perez GI, Chen Y, Blaser MJ (2010) Quantitation of major human cutaneous bacterial and fungal populations. J Clin Microbiol 48:3575-3581

9. Quince C, Curtis TP, Sloan WT (2008) The rational exploration of microbial diversity. ISME J 2:997-1006

10. Reeder J, Knight R (2009) The 'rare biosphere': a reality check. Nat Methods 6:636-637

11. Quince C, Lanzen A, Curtis TP, Davenport RJ, Hall N, Head IM, Read LF, Sloan WT (2009) Accurate determination of microbial diversity from 454 pyrosequencing data. Nat Methods 6:639-641

12. McLellan SL, Huse SM, Mueller-Spitz SR, Andreishcheva EN, Sogin ML (2010) Diversity and population structure of sewagederived microorganisms in wastewater treatment plant influent. Environ Microbiol 12:378-392

13. Huse SM, Welch DM, Morrison HG, Sogin ML (2010) Ironing out the wrinkles in the rare biosphere through improved OTU clustering. Environ Microbiol 12:1889-1898 
14. Palmer C, Bik EM, DiGiulio DB, Relman DA, Brown PO (2007) Development of the human infant intestinal microbiota. PLoS Biol 5:e177

15. Smillie CS, Smith MB, Friedman J, Cordero OX, David LA, Alm EJ (2011) Ecology drives a global network of gene exchange connecting the human microbiome. Nature 480:241-244

16. Sokol H, Pigneur B, Watterlot L, Lakhdari O, Bermudez-Humaran LG, Gratadoux JJ, Blugeon S, Bridonneau C, Furet JP, Corthier G, Grangette C, Vasquez N, Pochart P, Trugnan G, Thomas G, Blottiere HM, Dore J, Marteau P, Seksik P, Langella P (2008) Faecalibacterium prausnitzii is an anti-inflammatory commensal bacterium identified by gut microbiota analysis of Crohn disease patients. Proc Natl Acad Sci U S A 105:16731-16736

17. Angiuoli SV, Matalka M, Gussman A, Galens K, Vangala M, Riley DR, Arze C, White JR, White O, Fricke WF (2011) CloVR: a virtual machine for automated and portable sequence analysis from the desktop using cloud computing. BMC Bioinformatics 12:356

18. Meyer F, Paarmann D, D'Souza M, Olson R, Glass EM, Kubal M, Paczian T, Rodriguez A, Stevens R, Wilke A, Wilkening J, Edwards RA (2008) The metagenomics RAST server - a public resource for the automatic phylogenetic and functional analysis of metagenomes. BMC Bioinformatics 9:386

19. Caporaso JG, Kuczynski J, Stombaugh J, Bittinger K, Bushman FD, Costello EK, Fierer N, Pena AG, Goodrich JK, Gordon JI, Huttley GA, Kelley ST, Knights D, Koenig JE, Ley RE, Lozupone CA, McDonald D, Muegge BD, Pirrung M, Reeder J, Sevinsky JR, Turnbaugh PJ, Walters WA, Widmann J, Yatsunenko T, Zaneveld J, Knight R (2010) QIIME allows analysis of high-throughput community sequencing data. Nat Methods 7:335-336

20. Caporaso JG, Lauber CL, Walters WA, Berg-Lyons D, Huntley J, Fierer N, Owens SM, Betley J, Fraser L, Bauer M, Gormley N, Gilbert JA, Smith G, Knight R (2012) Ultra-high-throughput microbial community analysis on the Illumina HiSeq and MiSeq platforms. ISME J. doi:10.1038/ismej.2012.8

21. Dominguez-Bello MG, Costello EK, Contreras M, Magris M, Hidalgo G, Fierer N, Knight R (2010) Delivery mode shapes the acquisition and structure of the initial microbiota across multiple body habitats in newborns. Proc Natl Acad Sci U S A 107:11971-11975

22. Bisgaard H, Li N, Bonnelykke K, Chawes BL, Skov T, PaludanMuller G, Stokholm J, Smith B, Krogfelt KA (2011) Reduced diversity of the intestinal microbiota during infancy is associated with increased risk of allergic disease at school age. J Allergy Clin Immunol 128(646-652):e641-e645

23. Bik EM, Long CD, Armitage GC, Loomer P, Emerson J, Mongodin EF, Nelson KE, Gill SR, Fraser-Liggett CM, Relman DA (2010) Bacterial diversity in the oral cavity of 10 healthy individuals. ISME J 4:962-974

24. Jernberg C, Lofmark S, Edlund C, Jansson JK (2010) Long-term impacts of antibiotic exposure on the human intestinal microbiota. Microbiology 156:3216-3223

25. Dethlefsen L, Huse S, Sogin ML, Relman DA (2008) The pervasive effects of an antibiotic on the human gut microbiota, as revealed by deep 16S rRNA sequencing. PLoS Biol 6:e280

26. Dethlefsen L, Relman DA (2011) Incomplete recovery and individualized responses of the human distal gut microbiota to repeated antibiotic perturbation. Proc Natl Acad Sci U S A 108(Suppl 1):4554-4561

27. de Lastours V, Chau F, Tubach F, Pasquet B, Ruppe E, Fantin B (2010) Independent behavior of commensal flora for carriage of fluoroquinolone-resistant bacteria in patients at admission. Antimicrob Agents Chemother 54:5193-5200

28. Lee HH, Molla MN, Cantor CR, Collins JJ (2010) Bacterial charity work leads to population-wide resistance. Nature 467:82-85

29. Sommer MO, Dantas G, Church GM (2009) Functional characterization of the antibiotic resistance reservoir in the human microflora. Science 325:1128-1131
30. Rhoads DD, Wolcott RD, Percival SL (2008) Biofilms in wounds: management strategies. J Wound Care 17:502-508

31. Wolcott RD, Rhoads DD (2008) A study of biofilm-based wound management in subjects with critical limb ischaemia. J Wound Care 17:145-148, 150-142, 154-145

32. Wolcott RD, Rhoads DD, Dowd SE (2008) Biofilms and chronic wound inflammation. J Wound Care 17:333-341

33. Dowd SE, Sun Y, Secor PR, Rhoads DD, Wolcott BM, James GA, Wolcott RD (2008) Survey of bacterial diversity in chronic wounds using pyrosequencing, DGGE, and full ribosome shotgun sequencing. BMC Microbiol 8:43

34. Hall-Stoodley L, Costerton JW, Stoodley P (2004) Bacterial biofilms: from the natural environment to infectious diseases. Nat Rev Microbiol 2:95-108

35. Wolcott RD, Rumbaugh KP, James G, Schultz G, Phillips P, Yang Q, Watters C, Stewart PS, Dowd SE (2010) Biofilm maturity studies indicate sharp debridement opens a time-dependent therapeutic window. J Wound Care 19:320-328

36. Dowd SE, Sun Y, Smith E, Kennedy JP, Jones CE, Wolcott R (2009) Effects of biofilm treatments on the multi-species Lubbock chronic wound biofilm model. J Wound Care 18 (508):510-512

37. Wolcott RD, Gontcharova V, Sun Y, Zischakau A, Dowd SE (2009) Bacterial diversity in surgical site infections: not just aerobic cocci any more. J Wound Care 18:317-323

38. Wolcott RD, Gontcharova V, Sun Y, Dowd SE (2009) Evaluation of the bacterial diversity among and within individual venous leg ulcers using bacterial tag-encoded FLX and titanium amplicon pyrosequencing and metagenomic approaches. BMC Microbiol 9:226

39. Dowd SE, Delton Hanson J, Rees E, Wolcott RD, Zischau AM, Sun Y, White J, Smith DM, Kennedy J, Jones CE (2011) Survey of fungi and yeast in polymicrobial infections in chronic wounds. $\mathrm{J}$ Wound Care 20:40-47

40. Bizzini A, Durussel C, Bille J, Greub G, Prod'hom G (2010) Performance of matrix-assisted laser desorption ionization-time of flight mass spectrometry for identification of bacterial strains routinely isolated in a clinical microbiology laboratory. J Clin Microbiol 48:1549-1554

41. Degand N, Carbonnelle E, Dauphin B, Beretti JL, Le Bourgeois M, Sermet-Gaudelus I, Segonds C, Berche P, Nassif X, Ferroni A (2008) Matrix-assisted laser desorption ionization-time of flight mass spectrometry for identification of nonfermenting gramnegative bacilli isolated from cystic fibrosis patients. J Clin Microbiol 46:3361-3367

42. Mellmann A, Cloud J, Maier T, Keckevoet U, Ramminger I, Iwen P, Dunn J, Hall G, Wilson D, Lasala P, Kostrzewa M, Harmsen D (2008) Evaluation of matrix-assisted laser desorption ionizationtime-of-flight mass spectrometry in comparison to $16 \mathrm{~S}$ rRNA gene sequencing for species identification of nonfermenting bacteria. $\mathrm{J}$ Clin Microbiol 46:1946-1954

43. Seng P, Drancourt M, Gouriet F, La Scola B, Fournier PE, Rolain JM, Raoult D (2009) Ongoing revolution in bacteriology: routine identification of bacteria by matrix-assisted laser desorption ionization time-of-flight mass spectrometry. Clin Infect Dis 49:543-551

44. van Veen SQ, Claas EC, Kuijper EJ (2010) High-throughput identification of bacteria and yeast by matrix-assisted laser desorption ionization-time of flight mass spectrometry in conventional medical microbiology laboratories. J Clin Microbiol 48:900-907

45. Stevenson LG, Drake SK, Shea YR, Zelazny AM, Murray PR (2010) Evaluation of matrix-assisted laser desorption ionization-time of flight mass spectrometry for identification of clinically important yeast species. J Clin Microbiol 48:3482-3486 